\title{
Shapley-like values without symmetry
}

\author{
Jacob North Clark* \\ Stephen Montgomery-Smith ${ }^{\dagger}$
}

\begin{abstract}
Following the work of Lloyd Shapley on the Shapley value, and tangentially the work of Guillermo Owen, we offer an alternative non-probabilistic formulation of part of the work of Robert J. Weber in his 1978 paper "Probabilistic values for games." Specifically, we focus upon efficient but not symmetric allocations of value for cooperative games. We retain standard efficiency and linearity, and offer an alternative condition, "reasonableness," to replace the other usual axioms. In the pursuit of the result, we discover properties of the linear maps that describe the allocations. This culminates in a special class of games for which any other map that is "reasonable, efficient" can be written as a convex combination of members of this special class of allocations, via an application of the Krein-Milman theorem.
\end{abstract}

\section{Introduction}

\section{$1.1 \quad$ History and Applications}

\subsubsection{The initial work}

In Shapley's 1953 work, entitled "A value for n-person games.", Lloyd Shapley established an important idea in the theory of collaborative games. In Shapley's own words, "the possibility of evaluating games is therefore of critical importance." A player in the game needs to know their prospects, what they might receive compared to what they might produce on their own. Shapley's work set forth an axiomatically based way to do just that.

\subsubsection{Applications and iterations}

Since its first appearance, the Shapley value has been utilized in numerous contexts.

One context in which the Shapley value appears is in social network analysis. A person or organization might want to know who is the most important, or most influential in a network. In social contexts, one might want to impartially find the leader of a community or

\footnotetext{
${ }^{*}$ Department of Mathematics, University of Missouri-Columbia, jnclark@mail.missouri.edu

${ }^{\dagger}$ Department of Mathematics, University of Missouri-Columbia, stephen@missouri.edu Mathematics subject classification: 91A12, 52A20

Key words and phrases: Shapley value, cooperative game, reasonable, efficient
} 
rank the importance of members of a team. In a strictly economic sense, this could be used to target demonstrations or free samples of products, or used to target advertising dollars to the "taste makers" of a network. More detail regarding these ideas can be found in the work of Gómez et al., Narayanam and Narahari, Papapetrou et al. [8, 13, 15], and more generally the seminal work of Myerson [12] on "Graphs and Cooperation in Games".

Additionally, the Shapley value has been used in more general economic and political applications. Mertens has a compact writeup, "Some Other Economic Applications of the Value" [10] which discussed some of these applications, such as taxation and redistribution, and economies with fixed prices. Additionally, for voting games, the Shapley-Shubik power index builds on the ideas of the Shapley value to measure the power of each vote in voting games [17], something of interest to the field of political science among other fields.

In many, if not all, cases in a usable context, the computations necessary to calculate this information are numerous, if not computationally prohibitive. As such, many approximation schemes have appeared, as seen in the papers by Owen, Fatima et al. and Castro et al. in 1971/72, 2008 and 2009 respectively [14, 7, 3]. Algorithms using linear and polynomial techniques have been considered, among others.

With all of this activity, one might question Shapley's initial axioms. What is the fairness that his axioms describe? There have been many explorations of variations of the Shapley value concept, such as probabilistic values and indices of power as summarized in "Variations on the shapley value" of Monderer and Samet [11]. What happens when an axiom is weakened or removed? In Weber's paper of 1988, "Probabilistic values for games," there was an initial investigation of some of these ideas for the probabilistic value view of the Shapley value. For other explorations of Weber's work, Derks [6] offers another proof.

This paper covers some of the same ideas as the above with alternative assumptions placed on the allocations, aiming to make apparent the intermediary implications of the work, as well as offering another method of proof for the main result.

\subsubsection{Contributions of the paper}

In the paper [18], Weber gave many results on the theory of probabilistic values for games. One in particular is the fact that one can characterize games that are efficient without symmetry, i.e. random order values, as probabilistic values [18, Theorems 12 and 13]. In this paper, we offer an alternative idea and path to the results in the world of these non-symmetric games, focusing upon intermediary results, with the results below.

Note. These results were inspired by the papers $[14,16]$ and without knowledge of $[18,5]$, until later on in idea development. The main difference between this paper, and the one of Weber is we begin with more restricted, but reasonable assumptions, the Krein-Millman theorem is used in the proof of the main result, and properties of allocations themselves are looked into individually, rather than the whole process at once. Our process also differs from the presentation of [5], in our focus upon the matrices and the Krein-Millman theorem as a vehicle to understand the process. 


\subsection{Review \& Definitions}

To begin, we must first familiarize ourselves with the notion of an $n$-person cooperative game in the style of Shapley [16], or for a more modern presentation see the exposition of Maschler et al. [9]. In this section, and the ones following, a cooperative game can be characterized as the following sections describe.

\subsubsection{Characteristic functions of games}

We begin with a set of players $N=\{1,2,3,4, \ldots, n\}$, who may or may not be cooperating with one another. For convenience, we denote games with this number of players $|N|$-player games, or more commonly $n$-player games. With our set of players, we now endeavor to find a convenient way to mathematically express the possible gains that various subsets of players would receive if they collaborated. To accomplish this, we utilize characteristic functions.

Definition 1.1. Given an $n$-player cooperative game, with players coming from the set $N$, we characterize the game in terms of possible collaborations, via its characteristic function $v$, where $v: \mathbb{P}(N) \rightarrow \mathbb{R}^{\geq 0}$ or, alternatively the domain is $\{0,1\}^{|N|}$, i.e. in each situation, either a player is participating in a collaboration, or not, and the characteristic function assigns some value, or "gains" to this collaboration.

Now, we wish to obtain new information about these characteristic functions. First off, one may view them as a vector, with each entry in the vector corresponding to a member $T \in \mathbb{P}(N)$, applying some logical ordering scheme to the vector, such as increasing cardinality from the top to bottom of the vector. This vector view of a characteristic function will be useful in the considerations to come.

A characteristic function can exhibit several useful properties, described below.

Definition 1.2 (Monotonicity). A characteristic function $v$ is called monotone if given sets $S$ and $T$, with $S \subseteq T$, then $v(S) \leq v(T)$.

Definition 1.3 (Superadditivity). A characteristic function $v$ is called superadditive if for all $S, T \subset N$, if $S \cap T=\varnothing$, then $v(S \cup T) \geq v(S)+v(T)$.

\subsubsection{Shapley's value and the Collaborative Game}

With this information about the game, we now shift focus to that of allocating the spoils of the collaboration to each player. Typically, this solution is viewed as a vector, $\phi(N ; v)$ and the gains assigned to each player are denoted $\phi_{i}(N ; v)$ for player $i$. One can call this $\phi$ an allocation.

Definition 1.4. An allocation is a function $\phi$ with two inputs, the set of all players $N$, and a characteristic function $v$ with players from $N$, with the output of the function in $\mathbb{R}^{|N|}$.

The familiar Shapley value is one such allocation. To arrive at the Shapley value, we need to familiarize ourselves with his axioms for a "fair" solution $\phi$ to the problem of dividing spoils. For the following, let us assume $v(\varnothing)=0$. 
Axiom 1.1 (Efficiency). An allocation $\phi$ is efficient if for every coalitional game $(N ; v)$,

$$
\sum_{i \in N} \phi_{i}(N ; v)=v(N)
$$

Definition 1.5. Let $(N ; v)$ be a coalitional game, and let $i, j \in N$. Players $i$ and $j$ are symmetric if for every coalition $S \subseteq N \backslash\{i, j\}$, we have $v(S \cup\{i\})=v(S \cup\{j\})$.

Axiom 1.2 (Symmetry). An allocation $\phi$ is symmetric if for every coalitional game $(N ; v)$ and every pair of symmetric players $i$ and $j$ in the game: $\phi_{i}(N ; v)=\phi_{j}(N ; v)$

Definition 1.6. A player $i$ is called a null player in a game $(N ; v)$ if for every coalition $S \subseteq N$, including the empty coalition one has $v(S)=v(S \cup\{i\})$.

Axiom 1.3 (Null player property). An allocation $\phi$ satisfies the null player property if for every coalitional game $(N ; v)$ and every null player $i$ in the game, $\phi_{i}(N ; v)=0$.

Axiom 1.4 (Additivity). An allocation $\phi$ satisfies additivity if for every pair of coalitional games $(N ; v)$ and $(N ; w), \phi(N ; v+w)=\phi(N ; v)+\phi(N ; w)$.

Putting together all of our axioms, we can finally obtain the Shapley value.

Theorem 1.1 (Shapley value). There is a unique allocation $\phi_{i}(N ; v)$ satisfying efficiency, addativity, the null player property, and symmetry. This is the Shapley value.

Definition 1.7. The Shapley value is given by the equation

$$
\phi_{i}(N ; v)=\sum_{S \subseteq N \backslash\{i\}} \frac{|S| !(|N|-|S|-1) !}{|N| !}(v(S \cup\{i\})-v(S)) .
$$

The Shapley value can also be determined via a path integral calculation using a multilinear extension of $v$ as described by Owen [14]. This idea led, somewhat tangentially, to the formulation and results of this paper.

\subsubsection{Analysis background}

In the proofs of our results, we invoke several analytical results. So, to make the explanations clear, we present the results and concepts from functional analysis we shall draw from.

\subsubsection{Extreme Points}

We familiarize ourselves first with the concept of extreme points.

Definition 1.8. Let $X$ be a vector space, and suppose $K$ is a subset of $X$. A point $x \in K$ is an extreme point of $K$ if it does not lie on a line segment in $K$. To be more explicit, $x$ cannot be written as a (generalized) linear combination of distinct values in $K$.

We shall denote the set of extreme points of $K$ ex $(K)$. Typically, we consider convex $K$. Another way to view the definition of an extreme point $x$, following Bowers and Kalton [2], is if $u$ and $v$ are elements of $K$ such that $x=(1-t) u+t v$ for some $t \in(0,1)$, then $x=u=v$. Namely, we cannot write an extreme point as the convex combination of two distinct points in the set. 


\subsubsection{Metrizable topological vector spaces}

Following the exposition by Aliprantis and Border [1], we explore some facts about metrizable topological vector spaces, that will also be useful in proving our results. (Although, we do not need the full power of any of the statements.)

Definition 1.9. A neighborhood base at 0 is a collection of sets $\mathcal{B}$ of neighborhoods of 0 with the property that if $U$ is any neighborhood of 0 , there exists a $B \in \mathcal{B}$ such that $B \subset U$.

Theorem 1.2. A Hausdorff topological vector space is metrizable if and only if zero has a countable neighborhood base.

Theorem 1.3. In a complete metrizable locally convex space, the closed convex hull of a compact set is compact.

\subsubsection{The Krein-Milman Theorem}

The Krein-Millman Theorem, of functional analysis, is yet another result we shall utilize in our processes.

Theorem 1.4 (Krein-Milman). Suppose E is a locally convex Hausdorff topological vector space. If $K$ is a nonempty compact, convex subset of $E$, then

$$
K=\overline{c o}(e x K)
$$

where ex is the set of extreme points, and $\overline{c o}$ is the closed convex hull. In particular, ex $(K) \neq$ $\varnothing$

The proof of the Krein-Millman Theorem is non-constructive, however, the power of this result allows us to prove our results more intuitively.

\section{Allocations of Value}

\subsection{An introduction to allocations}

The Shapley value is a very specialized concept, and the given axioms might be too specific in some situations. From this point forward, we see if we can generalize the idea of division of total value $v(N)$ among the players in $N$, while reducing the number of required axioms. In addition, we shall see what properties we can determine based on these axioms.

The first thing we notice is the fact that these allocations $\phi$ can be viewed as linear maps, assuming we adopt Axiom 1.4 (Additivity). As such, they can be viewed as $|N| \times 2^{|N|}$ matrices. This view works quite well with the vector view of the characteristic functions discussed previously. Thus, we assume that Axiom 1.4 holds in all of our further considerations. It is helpful to note that in our considerations, $N$ is fixed, so $\phi$ can be viewed as a function of $v$ only. We also often make the identification between $\phi$ and its matrix counterpart. The majority of our results are proved using this identification. 
Inspired by the ideas presented by Owen [14], one can consider the path integrals along the edges of the region of integration, rather than the main diagonal (corresponding to the Shapley value). One may quickly see that the resulting allocations are well behaved. They can be defined via set chains of the players in $N$, specifically set chains that contain all players introduced one by one. More formally, allocations are "special" if they are the allocations described below.

Definition 2.1. A special allocation $\phi$ is an allocation that assigns marginal contributions directly to players in the following way. Given a set chain

$$
\varnothing=M_{0} \subset M_{1} \subset M_{2} \subset \ldots \subset M_{|N|-1} \subset M_{|N|}=N
$$

with $\left|M_{m+1} \backslash M_{m}\right|=1$, player $M_{m+1} \backslash M_{m}=\{i\}$ is assigned the "gains"

$$
\phi_{i}(N, v)=v\left(M_{m+1}\right)-v\left(M_{m}\right) .
$$

Thinking about this in matrix form we can see there is "special" structure here as well. Using the set chain made up of $M_{m}$, with $m=0$ to $m=|N|$ starting with $M_{0}=\varnothing$ with the restriction for all integer $m$ between 0 and $|N|-1$ that

$$
\left|M_{m+1} \backslash M_{m}\right|=1
$$

we see this means that we are adding a single player to $M_{j}$ at each step in the chain. This corresponds with the matrix of the allocation directly, namely for $m$ from 0 to $|N|-1$, we interpret the set chain as follows: in the row for player $M_{m+1} \backslash M_{m}=\{i\}$, we place a -1 in the column associated with $M_{m}$ and a 1 in the column associated with $M_{m+1}$. Looking at this, one might see why such allocations are "special".

We quickly see that there are nice consequences of viewing these special allocations as matrices. The sum of elements in the first column is -1 and the sum of elements in the last column is 1 . All other columns sum to 0 . The sum of the absolute value of the row elements is 2 , and additionally the sum of the absolute value of the interior column elements (not in the first or last column) is 2 as well. We will see all these consequences appear again, in more general context.

These chains are in turn in one to one correspondence with the set of all permutations of $|N|$ letters, which we can use to find the number of such allocations.

With this new terminology, we endeavor to build a representative set of allocations from which we can write any allocation. It turns out, we can do so for so-called "reasonable, efficient" allocations, and it turns out that this set of representatives is the set of all the special allocations.

\subsubsection{Efficiency in allocations}

We offer a slight generalization of Shapley's efficiency useful to out situation to begin

Axiom 2.1. An allocation $\phi$ with matrix $A$ to be efficient if

$$
\sum_{i=1}^{n} \phi_{i}(N ; v)=v(N)-v(\varnothing)
$$

for all monotone $v$, where $\phi_{j}(N ; v)=A_{j} \cdot v$, with $A_{j}$ denoting the $j^{\text {th }}$ row of $A$. 
Note. For most practical applications, we can assume that $v(\varnothing)=0$. With this assumption, we will divide all the spoils, even those present when no work is done by any player, among those participating in the game. Thus, we could think of efficiency as

$$
\sum_{i=1}^{n} \phi_{i}(N ; v)=v(N)-v(\varnothing)=v(N) .
$$

This is of course closely related to Axiom 1.1 (Efficiency).

It turns out that the row-wise sum properties we observed in the special allocation's matrices are true of any efficient one.

Lemma 2.1. Column-wise, the sum of all the row elements in each column of the matrix of an efficient allocation is

$$
(-1,0, \ldots, 0,1)
$$

Proof. Suppose we have a $n$ player game, with set of players $N$. Let us also suppose we have an efficient allocation $\phi$, with matrix $A$. By definition,

$$
A \cdot v=\phi(N ; v)
$$

for all $v$, with $\phi_{j}(N ; v)$ being the allocation of value to each player. Taking this information, we can now multiply both sides of the equality by a row univector of length $n$, and obtain

$$
[1, \ldots, 1] A \cdot v=[1, \ldots, 1] \phi(N ; v) .
$$

Now, taking the right hand side, we notice

$$
[1, \ldots, 1] \phi(N ; v)=\sum_{j=1}^{n} \phi_{j}(N ; v) .
$$

Via efficiency,

$$
\sum_{j=1}^{n} \phi_{j}(N ; v)=v(N)-v(\varnothing)
$$

Of course,

$$
v(N)-v(\varnothing)=[-1,0, \ldots, 0,1] v .
$$

Putting all of this together,

$$
[1, \ldots, 1] A \cdot v=[-1,0, \ldots, 0,1] v .
$$

As this is true for all $v$, we obtain

$$
[1, \ldots, 1] A=[-1,0, \ldots, 0,1] .
$$

Therefore, the sum of the rows of $A$ is what we require.

Note. The converse of this result is trivially true, namely if the sum of all the row elements in each column is

$$
(-1,0, \ldots, 0,1)
$$

then the allocation is efficient. 


\subsubsection{Reasonableness in allocations}

We begin our study in earnest by by proposing a new axiom, "reasonableness".

Axiom 2.2. An allocation $\phi$ with matrix $A$ reasonable ${ }^{1}$ if for all monotone $v$,

$$
\min _{S: i \notin S}\{v(S \cup\{i\})-v(S)\} \leq A_{i} \cdot v=\phi_{i}(N ; v) \leq \max _{S: i \notin S}\{v(S \cup\{i\})-v(S)\},
$$

where the maximum and minimum are taken over all $S$, with $i \notin S$.

Why one might say this is "reasonable" is clear. A logical player in a game would not expect to get less than the smallest contribution they make to a group. In the same way, an impartial observer of a game would not expect a player to receive more than the maximum contribution a player made to any collaboration.

Lemma 2.2. Given a player $m$, there exists a monotone $v$ so

$$
\min _{S: m \notin S}\{v(S \cup\{m\})-v(S)\}=\max _{S: m \notin S}\{v(S \cup\{m\})-v(S)\} .
$$

i.e., the inequalities in Axiom 2.2 are equalities.

Proof. To begin, take $m$ to be a given player in your game. We wish to build a binary characteristic function (or simple game) $v_{m}$ so the minimum is equal to the maximum. We construct $v_{m}$ as follows: If $m \in S$ for each place, put 1 in that place, if not, place a 0 . By construction, this is monotone. Also by construction, the difference

$$
v_{m}(S \cup\{m\})-v_{m}(S)=1
$$

for all $S$ with $S \cap\{m\}=\varnothing$.

Note. This tells us $\phi_{m}\left(N ; v_{m}\right)=1$, and $\phi_{l}\left(N ; v_{m}\right)=0$ for $l \neq m$.

The next two results follow quickly from the definitions.

Lemma 2.3. The characteristic functions constructed in Lemma 2.2 are superadditive, as defined in Definition 1.3.

Proposition 2.4. The convex combination of two reasonable allocations is again reasonable.

Lemma 2.5. Given a matrix of a reasonable allocation $A$ with each row only containing a single -1 and a single 1 and the rest of the entries all being 0 , if a-1 falls in the column for a set $S$, then the associated 1 in that row must fall in a superset of $S, S \cup T$, where $|T|-|S \cap T|>0$.

\footnotetext{
${ }^{1}$ This condition clearly implies several other conditions sometimes used in the explorations of allocations, namely Weber's dummy axiom, and the null-player property (Axiom 1.3). Recall, the dummy axiom is a generalization of the null player property. A player $m$ is dummy in the game if

$$
v(S \cup\{i\})=v(S)+v(\{i\}) \text { for all } S \subset N \backslash\{i\} .
$$

The dummy axiom is simply if player $i$ is a dummy in the game $v$, then $\phi_{i}(v)=v(\{i\})$.
} 
Proof. Suppose to the contrary, we have an allocation matrix $A$, for which the ordering is in reverse, namely the -1 is in $S \cup T$ and 1 in $S$, in the row associated with player $j$. Build $v_{S}$ as the monotone function with $v_{S}(S)=0$ and $v_{S}(S \cup T)=1$ for all $T$ such that $S \cap T \neq \varnothing$. This function is monotone by construction. When we utilize our map $A$ to determine how to split the spoils, there is an immediate contradiction. For the player $j, \phi_{j}(v)=-1$. This contradicts the fact that reasonable allocations do not assign players negative spoils. At the very worst, for a monotone binary game $v$,

$$
0 \leq \phi_{i}(v, N) \leq 1
$$

which is non-negative.

\subsection{Extreme points of the reasonable efficient allocations}

By observation, with small sets of players one might infer that the special allocations are the set of extreme points for the reasonable, efficient allocations, see for example the appendices of [4] for some finite results. We now proceed to provide a proof of this assertion in general.

\subsubsection{Special allocations and sets}

To begin, recall we defined each special allocation based on a strictly increasing (by exactly one member at each step) chain of sets. For example, the following special allocation matrix for the game with 3 players,

$$
\left(\begin{array}{rrrrrrrr}
-1 & 1 & 0 & 0 & 0 & 0 & 0 & 0 \\
0 & -1 & 0 & 0 & 1 & 0 & 0 & 0 \\
0 & 0 & 0 & 0 & -1 & 0 & 0 & 1
\end{array}\right)
$$

ordered usually, as follows

$$
[\varnothing,\{1\},\{2\},\{3\},\{1,2\},\{1,3\},\{2,3\},\{1,2,3\}]^{t},
$$

is associated with the chain of sets

$$
\varnothing \subset\{1\} \subset\{1,2\} \subset\{1,2,3\}
$$

This idea of set chains and their connection to the special allocations will prove integral to our following arguments. Additionally, it allows us to see the cardinality of the set of all special allocations quite quickly, as we know that the number of these chains corresponds directly to the number of permutations of $n$ letters, that is $n !$.

To prove our result, we will suppose we have an extreme reasonable, efficient allocation not listed in the set of all special allocations. We must show this is impossible. To obtain this result, we must first note that any reasonable, efficient allocation has the following properties. 


\subsubsection{Structural constraints on reasonable efficient allocations}

Each reasonable, efficient allocation has many properties, as demonstrated previously, and shown explicitly in [4]. Via those requirements, we can find even more structure that will help us reach our result. To begin, we will find the row-wise paring of elements. To make the proof more clear, the following notation is introduced.

Definition 2.2. Each entry in the matrix of an allocation can be referred to by a player and set, given $A$, we denote each entry by

$$
A_{i, S}
$$

where $i$ denotes the player (row), as before, and $S$ denotes the column associated.

Note. Definition 2.2 allows us to prove things independent from the ordering of the sets making up the columns of our matrices. Thus, if we can prove the statement that follows for a single row, we have it for all rows.

This allows us to write the payout to any specific player simply, as

$$
\phi_{i}(v, N)=A_{i} \cdot v=\sum_{S \subset N} A_{i, S} v(S),
$$

recalling $A_{i}$ is the $i^{\text {th }}$ row of $A$, and the sum is taken over all $S \subset N$.

\subsubsection{Truncations of characteristic functions}

Definition 2.3. We call a set $S$ minimal in the sense of the characteristic function if $v(S)>$ 0 and there exists no set $T \subsetneq S$ with $v(T)>0$.

Definition 2.4. A truncation ${ }^{2}$ of a characteristic function $v$ is the characteristic function $w$ such that $w(S)=0$ for some minimal $S$, and $w(T)=v(T)$ for $T \neq S$.

Remark. We call $S$ the truncating set.

Lemma 2.6. If $v$ is monotone, then any truncation of $v$ is monotone.

Proof. Suppose $v$ is a monotone characteristic function. Then, we know $v(T) \geq v(S)$ for all $S \subseteq T$ by definition. Let us let $w$ be a truncation of $v$, with truncating set $S_{t}$. Recall, $v(T)=w(T)$ for all $T \neq S_{t}$, and our inequality stands without much work for the majority of our places. However, we must concern ourselves of the cases when $S_{t}$ appears, as $v\left(S_{t}\right)>$ $w\left(S_{t}\right)=0$. This is no obstacle for $T$ with $S_{t} \subset T$, as

$$
w(T)=v(T) \geq v\left(S_{t}\right)>w\left(S_{t}\right)
$$

and

$$
v\left(S_{t}\right)>w\left(S_{t}\right)=0=v(S)=w(S)
$$

for $S \subset S_{t}$. Recall, of course, the truncating set is minimal, and there are no subsets with $w(S)>0$. Therefore, any truncation of $v$ is again monotone.

\footnotetext{
${ }^{2}$ This is similar in spirit to Weber's deletion [18, Section 6]. The language truncation remains as we are thinking of these characteristic functions as vectors.
} 
Lemma 2.7. If $v$ is superadditive, then any truncation of $v$ is superadditive.

Proof. Suppose $v$ is a superadditive characteristic function. Then, we know $v(S \cup T) \geq$ $v(S)+v(T)$ for all $S$ and $T$ with $S \cap T=\varnothing$, by definition. Let us let $w$ be a truncation of $v$, with truncating set $S_{t}$. Recall, $v(T)=w(T)$ for all $T \neq S_{t}$, so the inequality stands without much work for the majority of our places. However, we must concern ourselves with the cases when $S_{t}$ appears, as $v\left(S_{t}\right)>w\left(S_{t}\right)=0$. Note, however

$$
w\left(S_{t} \cup T\right)=v\left(S_{t} \cup T\right) \geq v\left(S_{t}\right)+v(T)>w\left(S_{t}\right)+w(T)
$$

if $T \neq \varnothing$, and the statement is trivial if $T$ is empty. Therefore, any truncation of $v$ is again superadditive.

Definition 2.5. A pair truncation of the characteristic function $v$ is two successive truncations of $v$ with truncating sets $S$ and $S \cup\{p\}$ respectively, for a player $p$ with $p \notin S$.

Lemma 2.8. A pair truncation $w$ of a binary characteristic function $v$ with marginal contribution of player $p$ equal to 0 with truncating sets $S$ and $S \cup\{p\}$, where $S$ is any minimal set with $p \notin S$, retains the same 0 marginal contribution for $p$.

Proof. To begin, let us take a characteristic function $v$ with the marginal contribution of $p$ equal to 0 . Take the pairwise truncation of this $v$ with the truncating sets $S$ and $S \cup\{p\}$, as described, where $S$ is any minimal set with $p \notin S$. Recall for $v, v(S)$ and $v(S \cup\{p\})=1$.

Prior to pair truncation, we note that, for sets $T \cap\{p\}=\varnothing, v(T \cup\{p\})-v(T)=0$, as the marginal contribution of $p$ is 0 . Naturally, following the pair truncation, $w(T \cup\{p\})-w(T)$ is still equal to 0 for all sets $T$. For all the unchanged places, this is clear, and for the two changed places, rather than seeing $1-1=0$ for the case of $T=S$, our truncating set, we now observe $0-0=0$.

Note. This of course works on the characteristic functions one can construct following Lemma 2.2 for player $m$, and they all have the same (zero) marginal contribution for any player $p \in N \backslash\{m\}$.

\subsubsection{Extensions of characteristic functions}

Definition 2.6. Given a characteristic function $v_{M}$ on the set $M \subsetneq N$, we can extend it to a characteristic function $v_{N}$ on $N$ by setting

$$
v_{N}(S)=v_{M}(S \cap M) .
$$

Lemma 2.9. If a characteristic function $v_{M}$ on $M \subsetneq N$ is monotone, then its extension $v_{N}$ to $N$ is also monotone.

Proof. As $v_{M}$ is monotone, we have

$$
v_{M}(T) \geq v_{M}(S)
$$


for all $S$ and $T$ with $S \subset T$. For the extension, we note, as $S \subset T, S \cap M \subset T \cap M$

$$
\begin{aligned}
v_{N}(T) & =v_{M}(T \cap M) \\
& \geq v_{M}(S \cap M) \\
& =v_{N}(S)
\end{aligned}
$$

and therefore, the extension is also monotone.

Lemma 2.10. If a characteristic function $v_{M}$ on $M \subsetneq N$ is superadditive, then its extension $v_{N}$ to $N$ is also superadditive.

Proof. As $v_{M}$ is superadditive, we have

$$
v_{M}(S \cup T) \geq v_{M}(S)+v_{M}(T)
$$

for all $S$ and $T$ with $S \cap T=\varnothing$. For the extension, we note

$$
\begin{aligned}
v_{N}(S \cup T) & =v_{M}((S \cup T) \cap M) \\
& =v_{M}((S \cap M) \cup(T \cap M)) \\
& \geq v_{M}(S \cap M)+v_{M}(T \cap M) \\
& =v_{N}(S)+v_{N}(T),
\end{aligned}
$$

and thus, the extension is also superadditive.

Lemma 2.11. For the extensions of characteristic functions $M=N \backslash\{i\}$, the inequalities in the definition of reasonableness taken for player $i$ are equalities and

$$
\phi_{i}(N ; v)=0 .
$$

Proof. This is clear, as

$$
v(S \cup\{i\})=v(S)
$$

by the definition of an extension, and by the definition of reasonableness.

\subsubsection{Pairing behavior in the rows of a reasonable allocation}

In the following theorem, we establish a strong condition on the reasonable allocations, specifically their matrix counterparts. To our main result, this theorem serves as an integral component.

Theorem 2.12 (Pairing of row elements). Given a player (row) $i$ of a reasonable allocation's matrix A, the elements pair off in the following manner:

$$
A_{i, S}=-A_{i, S \cup\{i\}}
$$

for sets $S$ with $S \cap\{i\}=\varnothing$. 
Proof. To obtain this result, we consider a player $i$. (Any other player's information can be obtained identically.) Construct the following superadditive $v$, given $S$ with $S \cap\{i\}=\varnothing$

$$
v_{a}^{S}(T)= \begin{cases}1 & \text { if } S \subset T \\ 0 & \text { else }\end{cases}
$$

and

$$
v_{b}^{S}(T)= \begin{cases}1 & \text { if } S \subsetneq T \backslash\{i\} \\ 0 & \text { else. }\end{cases}
$$

Observe, $v_{a}^{S}$ is superadditive trivially, as

$$
v_{a}^{S}(Q \cup R) \geq v_{a}^{S}(Q)+v_{a}^{S}(R)
$$

for $Q$ and $R$ with $Q \cap R=\varnothing$. This can quickly be seen as $S \subset Q$ or $S \subset R$, but not both. So, at the very worst, $1 \geq 1+0$ or $1 \geq 0+1$.

Notice $v_{b}^{S}$ is a pair truncation of $v_{a}^{S}$, by construction, as it zeros out the $S$ and $S \cup\{i\}$ places precisely. Thus, $v_{b}^{S}$ is also superadditive. $v_{a}^{S}$ also has the property that the marginal contribution of player $i$ is 0 , observe, by construction, for $Q$ with $Q \cap\{i\}=\varnothing$,

$$
v_{a}^{S}(Q \cup\{i\})-v_{a}^{S}(Q)=\left\{\begin{array}{ll}
1-1=0 & \text { if } S \subset Q \\
0-0=0 & \text { if } S \not \subset Q
\end{array} .\right.
$$

Recall, $i \notin S$ by our initial choice of $S$. So, to obtain the pairing for $A_{i, S}$ and $A_{i, S \cup\{i\}}$, we observe

$$
\begin{aligned}
& \phi_{i}\left(N ; v_{a}^{S}\right)=A_{i} \cdot v_{a}^{S}=0 \\
& \phi_{i}\left(N ; v_{b}^{S}\right)=A_{i} \cdot v_{b}^{S}=0 .
\end{aligned}
$$

However, using this to our advantage, notice

$$
\begin{aligned}
0 & =\phi_{i}\left(N ; v_{a}^{S}\right)-\phi_{i}\left(N ; v_{b}^{S}\right) \\
& =A_{i} \cdot\left(v_{a}^{S}-v_{b}^{S}\right) \\
& =A_{i, S}+A_{i, S \cup\{i\}}
\end{aligned}
$$

Rearranging,

$$
A_{i, S}=-A_{i, S \cup\{i\}}
$$

as we wished. Repeating this process for all $S \subset N \backslash\{i\}$ gives us all the pairings we desire.

\subsubsection{Bounds on the matrix elements}

Lemma 2.13. Given a row $i$ of a reasonable allocation's matrix A, for nonempty $S$, with $S \cap\{i\}=\varnothing$

$$
0 \leq A_{i, S \cup\{i\}} \leq 1
$$

and

$$
0 \geq A_{i, S} \geq-1 \text {. }
$$

Namely, the non-negative entries fall in the columns associated with sets $S \cup\{i\}$ and the associated non-positive entries fall in the columns associated with $S$. 
Proof. Using techniques seen in the proof of Theorem 2.12, we can make short work of this statement. Consider

$$
v_{b}^{S}(T)= \begin{cases}1 & \text { if } S \subsetneq T \backslash\{i\} \\ 0 & \text { else }\end{cases}
$$

as seen previously, and

$$
v_{c}^{S}(T)= \begin{cases}1 & \text { if } S \subsetneq T \\ 0 & \text { else. }\end{cases}
$$

Note, $v_{c}^{S}$ is a truncation (not a pairwise truncation) of the $v_{a}^{S}$ from the superadditive proof of Theorem 2.12. Hence, it is superadditive. The only difference between $v_{b}^{S}$ and $v_{c}^{S}$ is $v_{b}^{S}(S \cup\{i\})=0$, while $v_{c}^{S}(S \cup\{i\})=1$. Recall, with $v_{b}^{S}$, the marginal contribution of player $i$ is always 0 . Note, also, for $v_{c}^{S}$, the marginal contribution of player $i$ falls between 0 and 1 , as the only difference from $v_{b}^{S}$ occurs at the place associated with $S$, which results in

$$
v_{c}^{S}(S \cup\{i\})-v_{c}^{S}(S)=1-0=1
$$

rather than 0 . Now, to use this to our advantage, we note

$$
\phi_{i}\left(N ; v_{b}^{S}\right)=A_{i} \cdot v_{b}^{S}=0 .
$$

and

$$
0 \leq \phi_{i}\left(N ; v_{c}^{S}\right)=A_{i} \cdot v_{c}^{S} \leq 1
$$

via reasonableness. However, there is little difference between $A_{i} \cdot v_{b}^{S}$ and $A_{i} \cdot v_{c}^{S}$, notice

$$
A_{i} \cdot v_{c}^{S}-A_{i} \cdot v_{b}^{S}=A_{i, S \cup\{i\}} .
$$

So, we may conclude

$$
\begin{gathered}
0 \leq \phi_{i}\left(N ; v_{c}^{S}\right)-\phi_{i}\left(N ; v_{b}^{S}\right) \leq 1 \\
0 \leq A_{i} \cdot v_{c}^{S}-A_{i} \cdot v_{b}^{S} \leq 1
\end{gathered}
$$

and utilizing Equation (3),

$$
0 \leq A_{i, S \cup\{i\}} \leq 1
$$

as we wished to show. By the pairings, we obtain

$$
0 \leq-A_{i, S} \leq 1,
$$

i.e.

$$
0 \geq A_{i, S} \geq-1
$$

again, as we wished to show.

Note. Via this lemma, we have the signs of nearly all of the matrix entries. The only missing are, for row $i A_{i, \varnothing}$ and $A_{i,\{i\}}$. This is covered by Lemma 2.14 below. 
Lemma 2.14. Given a reasonable, efficient allocation matrix $A$, we have $1 \geq A_{i,\{i\}} \geq 0$ for all players $i$, and hence, by the pairing $-1 \leq A_{i, \varnothing} \leq 0$.

Proof. We can utilize the information we have gained thus far to infer this information. First, recall that $-1 \leq A_{j,\{i\}} \leq 0$ for all $j \neq i$, via the pairings, as by the previous Lemma 2.13, $1 \geq A_{j,\{i, j\}} \geq 0$. More specifically,

$$
0 \geq \sum_{j \neq i} A_{j,\{i\}} \geq-1
$$

This is indeed the case, as if not, we shall reach a contradiction. Let us assume that

$$
\sum_{j \neq i} A_{j,\{i\}} \leq-1
$$

Via the pairings, we can follow each of the nonzero elements in the sum up the chain to $A_{j,\{i, j\}}$ and there must be a paired positive value there, for each $j$. Their sum, even though they might not remain in the same column remains more than 1 . In columns $\{i, j\}$ for each of these $j$, there must be a negative contribution to make the column-wise sum 0 . (It is possible there might be a splitting between two or more rows. Keep in mind no entry can exceed 1 in absolute value by Lemma 2.13 at this point, if we have any entry greater than 1 in absolute value, we have a contradiction. The sum, of course must remain 0.) Each of those entries pairs off with a superset in the same row. We can continue this process until we reach the set of all players $N$. In this column, we have the tail of every (possibly split up) chain we traveled along, and due to the pairing of the elements, the overall sum is greater than or equal to 1 (as each chain must carry at the very least all of its value along, even if it splits or combines along the way due to the pairings). This contradicts reasonableness, and thus we have that $\sum_{j \neq i} A_{j,\{i\}} \geq-1$ as we wished. Noting this, we see that it is imperative that $A_{i,\{i\}}$ must be greater than or equal to 0 and less than or equal to 1 , as, via efficiency, the sum of all elements in the column $\{i\}$ is 0 by Lemma 2.1. More explicitly,

$$
\sum_{j \neq i} A_{i,\{j\}}+A_{i,\{i\}}=0
$$

So

$$
A_{i,\{i\}}=-\left(\sum_{j \neq i} A_{i,\{j\}}\right)
$$

and

$$
0 \leq A_{i,\{i\}} \leq 1
$$

Clearly, as a result, $-1 \leq A_{i, \varnothing} \leq 0$ via the pairings.

So, we now have the signs for all of the row elements, and we can summarize our results in the following convenient way.

Theorem 2.15. Given a reasonable, efficent allocation with matrix A, for player $i$,

$$
\begin{array}{r}
1 \geq A_{i, S} \geq 0 \text { if } i \in S \\
-1 \leq A_{i, S} \leq 0 \text { if } i \notin S
\end{array}
$$


Proof. Refer to Lemmas 2.13 and 2.14.

We utilize some of the ideas found in the proof of Lemma 2.14 to get more general results, along with Theorems 2.12 and 2.15. So, as a consequence of these results, we can find another important fact for our reasonable, efficient allocations.

Lemma 2.16. Each column of a reasonable, efficient allocation matrix can contain no more than a sum of -1 of negative elements and a sum of 1 in positive elements.

Proof. Suppose the contrary, that there is a sum of more than -1 of the negative elements in one column. Our aim is to show this is not possible. Let us assume that this overflow of negatives occurs in the column $\{i, j\}$, in rows $l, \ldots$, and $m$, say. Now, via the pairing, we can follow each of these chains up, there must be a paired positive value in $A_{l,\{i, j, l\}}, \ldots$, and $A_{m,\{i, j, m\}}$ respectively, and their sum remains more than 1 . In columns $\{i, j, l\}, \ldots$, and $\{i, j, m\}$, there must be a negative contribution to make the column-wise sum 0 . (It is possible there might be a splitting between two or more rows. Keep in mind no entry can exceed 1 in absolute value, if we have any entry greater than 1 in absolute value, we have a contradiction. The sum, of course must remain 0.) Each of those entries pairs off with a superset in the same row. We can continue this process until we reach the set of all players $N$. In this column, we have the tail of every (possibly split up) chain we traveled along, and due to the pairing of the elements, the overall sum is greater than or equal to 1 (as each chain must carry all of its value along). This contradicts reasonableness.

The case of two or more positive values with sum greater than 1 is handled nearly identically, one just starts the chain at this point, noting the sum of the column must be 0 , and thus, there must be elements summing to greater than -1 in the column to compensate.

Therefore, we have what we set out to show, each column can contain no more than a sum of -1 of negative elements and a sum of 1 in positive elements.

Lemma 2.17. For a reasonable allocation $\phi$ with matrix $A$, the partial row-wise sum satisfies the equality

$$
\sum_{S: i \notin S} A_{i, S \cup\{i\}}=1
$$

Proof. Recall, from Lemma 2.2, we know there exists a $v_{i}$ for which

$$
\phi_{i}\left(N, v_{i}\right)=1
$$

by the squeezing of the reasonableness condition. This $v_{i}$ is precisely the one with a 1 in all places with $i \in T$. Now, noting that

$$
\phi_{i}\left(N, v_{i}\right)=A_{i} \cdot v_{i}=\sum_{S: i \notin S} A_{i, S \cup\{i\}},
$$

we obtain the result we desire.

Corollary (to Lemma 2.17). For a reasonable allocation $\phi$ with matrix $A$, the partial row-wise sum satisfies the equality

$$
\sum_{S: i \notin S} A_{i, S}=-1 .
$$


Remark. For a reasonable, efficient allocation with matrix $A$, the sum over all players $i$ of the elements $A_{i, S} \geq 0$ with cardinality of $S$ a fixed integer between 0 and $|N|$ is $\sum_{i} A_{i, S}=1 .{ }^{3} \triangleleft$

\subsection{Extreme points, and the reasonable efficient allocations}

In the journey to prove our results, we find the following lemma integral to our arguments as well.

Lemma 2.18. For an allocation $\phi$ with matrix $A$, if for all players $i$,

$$
A_{i, S} \text { is }\left\{\begin{array}{l}
\geq 0 \text { if } i \in S \\
\leq 0 \text { if } i \notin S
\end{array} .\right.
$$

and for $T$ with $T \cap\{i\}=\varnothing$

$$
A_{i, T}=-A_{i, T \cup\{i\}}
$$

and

$$
\sum_{T} A_{i, T \cup\{i\}}=1
$$

then the allocation $\phi$ is reasonable.

Proof. To begin, by Equations (7) and (8), we have that $\sum_{T} A_{i, T}=-1$, and all elements of the matrix are determined. To check for reasonableness, note

$$
\begin{aligned}
\phi_{i}(N ; v) & =A_{i} \cdot v \\
& =\sum_{S} A_{i, S} \cdot v(S) \\
& =\sum_{T} A_{i, T \cup\{i\}}(v(T \cup\{i\})-v(T))
\end{aligned}
$$

with $T \cap\{i\}=\varnothing$ by our map and Equation (7). By Equations (6) and (8), we note that $\phi_{i}(N ; v)$ is a generalized linear combination of the marginal contributions of each player, as all the elements in the sum are greater than or equal to 0 and sum to 1 . Trivially, a generalized convex combination lies between the

$$
\min _{T: i \notin T}\{v(T \cup\{i\})-v(T)\} \text { and } \max _{T: i \notin T}\{v(T \cup\{i\})-v(T)\} .
$$

Thus, the allocation is reasonable, as required.

Lemma 2.19. The converse to Lemma 2.18 is also true.

Proof. For the reverse, suppose matrix $A$ and its associated $\phi$ is reasonable. In part, this now equates to showing that the only possible choices for elements satisfy Equations (6) to (8). The pairings, we note, can be found utilizing the results of Theorem 2.12. Identically, the partial row sum in Equation (7) is obtained via Lemma 2.17. Both of these theorems use

\footnotetext{
${ }^{3}$ This is similair to the implicit result alluded to in the proof of [18, Theorem 13$]$.
} 
only the satisfaction of the reasonability condition in the body of their results. Taking note that we have the pairings, we can obtain almost all the signs we wish via Lemma 2.13. However, in our proofs above, we cannot obtain the signs of $A_{i, \varnothing}$ and $A_{i,\{i\}}$ without efficiency. However, if we allow ourselves to utilize non-superadditive, yet monotone vectors, we can get the remaining signs we wish. To prove $A_{i, \varnothing} \leq 0$ for all players $i$, one can observe, via multiplying the map $A$ by the characteristic function

$$
v=[1,1,1, \ldots, 1,1,1]^{t}
$$

focusing on the $i^{\text {th }}$ row,

$$
A_{i, \varnothing}+A_{i,\{1\}}+A_{i,\{2\}}+\cdots+A_{i,\{1,2\}}+\cdots+A_{i, N \backslash\{n\}}+\cdots+A_{i, N \backslash\{1\}}+A_{i, N}=0
$$

by reasonableness. Similarly, we obtain

$$
1 \geq A_{i,\{1\}}+A_{i,\{2\}}+\cdots+A_{i,\{1,2\}}+\cdots+A_{i, N \backslash\{n\}}+\cdots+A_{i, N \backslash\{1\}}+A_{i, N} \geq 0
$$

by using the definition of reasonableness, Axiom 2.2, along with the vector

$$
v=[0,1,1, \ldots, 1,1,1]^{t}
$$

This is evident due to the fact that each player must receive no less than 0 and no more than 1 , based on the marginal contribution bounds, given the fact our characteristic function is monotone. Combining these two facts, one quickly sees that

$$
-1 \leq A_{i, \varnothing} \leq 0
$$

as required. $1 \geq A_{i,\{i\}} \geq 0$ is immediately picked up via the pairings. Therefore, we have the last piece of needed info, Equation (6).

Lemma 2.20 (Extreme points of the reasonable, efficient allocations). The extreme points of the set of all reasonable, efficient allocations are contained within the set of special allocations.

Proof. We prove this using the matricies of the allocations. Suppose first we have a reasonable, efficient allocation matrix $A$ that is an extreme point, but is not a member of the set of all special allocations. Our aim is to reach a contradiction. To begin, via efficiency, and Lemma 2.1, we know there is at least one entry in the first column that is negative, in the $i^{\text {th }}$ row, say. Starting at this entry, we build a set chain as introduced in Section 2.2.1 by utilizing the row-wise pairings of Theorem 2.12. Given our choice of negative element in the first column, $A_{i, \varnothing}$, by the pairings, we know there is a positive, and equal in absolute value, entry in $A_{i,\{i\}}$. Calling upon efficiency yet again, for the internal columns, we know that the sum of all the entries is equal to 0 . Thus, there exists at least one negative element in row $j$, say, $A_{j,\{i\}}$. This entry in turn has a paired entry in a superset $\{i, j\}, A_{j,\{i, j\}}$. Continuing this process, we can continue to build a set chain to represent this path through the allocation. The set chain would appear as something of the form

$$
\varnothing \subset\{i\} \subset\{i, j\} \subset\{i, j, k\} \subset \cdots \subset N .
$$


If, at all stages the at least one player was exactly one player, we have a contradiction. Recalling Lemma 2.16, each column can contain no more than a sum of -1 in negative elements and a sum of 1 in positive elements. If there was only a single choice in each case, as the sum of the first column must be -1 , that forces $A_{i, \varnothing}=-1$. In turn, $A_{i,\{i\}}=1$. Continuing, it must be the case that $A_{j,\{i\}}=-1$. Following this along the chain, we know every element we touched was either a -1 or 1 by the pairings and efficiency (Lemma 2.1). More precisely, it was a special allocation already, a contradiction. Therefore, we know that at in at least one instance when we were building our chain, we had two choices of elements (either both positive, or both negative, distinct from the element we started with), in row $l$ and $m$, say. If, in our initial chain, we chose row $l$, we can make a secondary set chain by choosing row $m$ at the juncture and following this alternative path. More explicitly, for some $T$ with $T \cap\{l, m\}=\varnothing$ and $l \neq m$, our two set chains would contain the links

$$
T \subset T \cup\{l\}
$$

for the first set chain, and

$$
T \subset T \cup\{m\}
$$

for the second set chain, respectively. Thus, we have two distinct set chains. From these two distinct set chains, we can find the associated special allocation matrices $S^{a}$ and $S^{b}$, say. These special allocations will be used to demonstrate that our assumed extreme $A$ is not. Following the same ideas we did in the case of the characteristic functions, we wish to find a way to modify our $A$ in small ways on either side, both modified matrices still reasonable, with a convex combination of the matrices equal to $A$ itself. To do this, we choose an $\epsilon$ in the following way:

$$
\epsilon<\min \left\{\begin{array}{l}
A_{M_{m+1} \backslash M_{m}, M_{m+1}} \\
1-A_{M_{m+1} \backslash M_{m}, M_{m+1}} \quad A_{M_{m+1} \backslash M_{m}, M_{m+1}} \neq 1
\end{array}\right.
$$

where $M_{m}$ and $M_{m+1}$ are consecutive elements in the set chains we have defined above. First, notice $A_{M_{m+1} \backslash M_{m}, M_{m+1}}>0$ by construction. Notice also, as we have assumed $A$ is not a special allocation, and have excluded the entries $A_{M_{m+1} \backslash M_{m}, M_{m+1}} \neq 1$ from consideration in $\epsilon$, we have $0<\epsilon<1$. By this choice of $\epsilon$, we claim that one can both add and subtract $\epsilon S^{a} \pm \epsilon S^{b}$ without compromising the reasonableness of the map. To see this, consider

$$
A \pm\left(\epsilon S^{a}-\epsilon S^{b}\right)
$$

alongside Lemma 2.18. We note by our choice of $\epsilon$, the signs of each element of $A \pm\left(\epsilon S^{a}-\epsilon S^{b}\right)$ are unchanged. Trivially, we satisfy the pairings for $T$ with $T \cap\{i\}=\varnothing$ as $A, S^{a}$ and $S^{b}$ do as well, and addition and subtraction of matrices with the pairings produces other matrices satisfying the pairings. Finally, we note that each row in $\pm\left(\epsilon S^{a}-\epsilon S^{b}\right)$ makes a contribution of 0 to the row sum of $A^{\prime}=A \pm\left(\epsilon S^{a}-\epsilon S^{b}\right)$, and the sum $\sum_{T} A_{i, T \cup\{i\}}^{\prime}=1$, as, in net, all that is done is an addition and subtraction of $\epsilon$ to the sum. Thus, all of the requirements of Lemma 2.18 are fulfilled, and we can conclude that $A^{\prime}$ is reasonable. Clearly, as $A, S^{a}$ and $S^{b}$ are efficient, the sum $A^{\prime}=A \pm\left(\epsilon S^{a}-\epsilon S^{b}\right)$ is efficient as well utilizing Lemma 2.1 and its converse. Thus, we have two additional derived reasonable, efficient maps, $A_{a}$ and $A_{b}$, with

$$
\begin{aligned}
& A_{a}=A-\left(\epsilon S^{a}-\epsilon S^{b}\right) \\
& A_{b}=A+\left(\epsilon S^{a}-\epsilon S^{b}\right) .
\end{aligned}
$$


Notice

$$
A=\frac{1}{2} A_{a}+\frac{1}{2} A_{b} .
$$

This contradicts the assumed extremeness of $A$. Therefore, we can conclude that the extreme points of the set of all reasonable, efficient allocations are contained within the set of special allocations.

Note, it is clear from the definitions that each special allocation is an extreme point. So, as a result, we may state the following.

Theorem 2.21. The extreme points of the set of all reasonable, efficient allocations are precisely the special allocations.

\subsection{Reasonable efficient allocations and the convex hull of the spe- cial allocations}

With all of the machinery we have established, we can now prove a nice property of the special allocations, namely any reasonable, efficient allocation can be written as a positive (generalized) linear combination of the special allocations. We now prove the main result.

Theorem 2.22. Any reasonable, efficient allocation can be written as a convex combination of the special allocations, more strongly,

An allocation is reasonable and efficient if and only if the allocation lies within the convex hull of the special allocations.

Proof. We prove this for the matrix $A$ of an allocation $\phi$. If $A$ lies within the convex hull of all special allocation matrices, it is clear that our proposition is true based on the inherent properties of special allocations explored in Proposition 2.4, in particular.

Conversely, suppose we have the set of all reasonable, efficient allocations, $\mathcal{R}$, say. Our goal is to show that $\mathcal{R}$ is identical to the convex hull of the special allocations. First, we know that the set of all reasonable, efficient allocations is compact, as $\mathcal{R}$ is finite dimensional over $\mathbb{R}$, and each entry of the matrix of a reasonable, efficient allocation is bounded (by -1 and 1 inclusive via Theorem 2.15.) As a result, we have a closed and bounded set, and under our conditions, this results in a compact set via the Heine-Borel theorem. From our prior exploration in Proposition 2.4, we know that $\mathcal{R}$ is convex. Via Lemma 2.20, we know that the extreme points of $\mathcal{R}$ are precisely the set of all special allocations, $\mathcal{S}$, say. Putting these facts together, we may conclude, by Theorem 1.4 (Krein-Milman) that

$$
\begin{aligned}
\mathcal{R} & =\overline{\mathrm{co}}(\operatorname{ex} \mathcal{R}) \\
& =\overline{\mathrm{co}}(\mathcal{S})
\end{aligned}
$$

This is nearly what we wish to show. Note, $\mathcal{S}$ is a finite set and is also bounded, hence closed, thus one can make the last conclusion, that the closure of the convex hull is the convex hull itself, via the theorems and definitions in Section 1.2.5. Therefore,

$$
\begin{aligned}
\mathcal{R} & =\overline{\mathrm{co}}(\mathcal{S}) \\
& =\operatorname{co}(\mathcal{S}),
\end{aligned}
$$


and we now have what we set out to prove. Any reasonable, efficient allocation lies within the convex hull of the special allocations.

We now have both directions of our proof, and so, we can think of the special allocations as a spanning set of sorts for the set of reasonable, efficient allocations. More specifically, any such allocation is in the cone made up of the special allocations. Viewing things this way lets us prove several more results.

\subsection{Consequences of the main result}

With the knowledge gained through Theorem 2.22, we can now prove even more properties of the reasonable, efficient allocations.

Theorem 2.23. Given a reasonable, efficient allocation matrix A, the sum of each interior column (not first or last) in absolute value is 2 .

Proof. This is trivial to see in the case of the special allocation matrices. To obtain the general result, we must see that the result holds for a convex combination of two reasonable, efficient allocations that satisfy the condition already. ${ }^{4}$ Observe, the column-wise sum, of two such allocation matrices, $P$ and $Q$ for column $S$. $\left(P_{1, S}, P_{2, S}, \ldots, P_{n-1, S}, P_{n, S}\right)^{t}$ and, similarly $\left(Q_{1, S}, Q_{2, S}, \ldots, Q_{n-1, S}, Q_{n, S}\right)^{t}$. By our assumption,

$$
\begin{aligned}
& \sum_{i=1}^{n}\left|P_{i, S}\right|=2 \\
& \sum_{i=1}^{n}\left|Q_{i, S}\right|=2
\end{aligned}
$$

for $S \neq \varnothing$ and $S \neq N$. Now, taking the convex combination of the columns, we obtain

$$
\left(t P_{1, S}+(1-t) Q_{1, S}, \ldots, t P_{n, S}+(1-t) Q_{n, S}\right)^{t} .
$$

We note

$$
\begin{aligned}
\sum_{i=1}^{n}\left|t P_{i, S}+(1-t) Q_{i, S}\right| & \leq \sum_{i=1}^{n} t\left|P_{i, S}\right|+(1-t)\left|Q_{i, S}\right| \\
& =t \sum_{i=1}^{n}\left|P_{i, S}\right|+(1-t) \sum_{i=1}^{n}\left|Q_{i, S}\right| \\
& =t \cdot 2+(1-t) \cdot 2 \\
& =2
\end{aligned}
$$

by the triangle inequality and properties of the absolute value. Now, one must argue that this inequality is necessarily an equality. Recall, the triangle inequality is an equality if both numbers are non-positive or non-negative. By Lemma 2.13 we see that $P_{i, S}$ and $Q_{i, S}$ necessarily are both non-positive or non-negative. So the triangle inequality is a triangle equality, and we have the equality we desire.

\footnotetext{
${ }^{4}$ As any reasonable allocation can be written as a (generalized) convex combination, this gives us our result.
} 
Theorem 2.24. The sum of each row of an allocation's matrix in absolute value is 2 .

Note. We could have proved this without our main result, however, it is presented here with similar in spirit results.

Proof. Recall, via Lemma 2.13, the elements summed in Lemma 2.17 are all non-negative. Similarly, the sum in the corollary is of all non-positive numbers. As a result,

$$
\sum_{S: i \notin S}\left|A_{i, S \cup\{i\}}\right|=1
$$

and

$$
\sum_{S: i \notin S}\left|A_{i, S}\right|=1 .
$$

Hence,

$$
\sum_{S}\left|A_{i, S}\right|=2 .
$$

Remark. We can now recognize "un-reasonable" allocations quite easily. If the row sums of the absolute value of the elements of the matrix of the allocation are not equal to 2 and if the column sums of an interior column are not equal to 2 , we can immediately notice it is unreasonable.

\section{Consequential Parallel Results}

With slightly different assumptions, we can get the same results, in a broader context.

\subsection{Superadditivity as a replacement for monotonicity}

To get similair results for superadditive functions, we need only add the following axioms,

Axiom 3.1. The value of $v(\varnothing)=0$.

Remark. This is of course true for superadditve functions, and is not so much an axiom, but a consequence of the definition of superadditivity. If $v(\varnothing)>0$, we reach a contradiction, as $v(S)=v(S \cup \varnothing) \geq v(S)+v(\varnothing)$.

Typically, one would assume Axiom 3.1, if one wants to divide all "produced" among the players of the game. This was mentioned when we first defined efficiency in Axiom 2.1.

Axiom 3.2. The sum of all elements in each row is of the matrix of the allocation $\phi$ is 0 .

Note. The choice of the first column is arbitrary, due to the fact that $v(\varnothing)=0$ via Axiom 3.1, or its following remark. Thus, without loss of generality, Axiom 3.2 always holds, as we can pick the value in the first column to make the sum work. 
To first proceed, let us get an idea what we can do with superadditive characteristic functions.

Proposition 3.1. The set of all superadditive binary characteristic functions (superadditive simple games) form a spanning set for the monotone binary characteristic function with $v(\varnothing)=0$.

Proof. If we look at the linear span of superadditive binary $v$, they do form a spanning set for all monotone $v$ with $v(\varnothing)=0$. We can see this by viewing them in the following way. Consider all of the "monotone" set chains,

$$
\varnothing \subset\{i\} \subset\{i, j\} \subset\{i, j, k\} \subset \ldots \subset N,
$$

adding a single player in each subsequent set in the chain. This set chain, can of course be associated with a special allocation, but it can also be associated with a superadditive characteristic function $v_{s a},{ }^{5}$ following the steps below. Place a 1 in all of the places associated with each non-empty set in the chain, and a 0 in all others. This is trivially superadditive, as

$$
v_{s a}(S)+v_{s a}(T) \leq v_{s a}(S+T)
$$

by construction for $S \cap T=\varnothing$. Notice also, we can truncate this $v_{s a}$, starting the assignment of ones at any point midway through the set chain still yields a superadditive characteristic function. Recall, superadditive implies monotone in our considerations. However, the reverse direction is easily seen to be false, there are certainly monotone characteristic functions that are not superadditive. To see that these are within the linear span of the superadditive characteristic functions, we can follow a constructive process detailed below. Given a monotone $v$, there is at least one set with smallest cardinality. Take all of the chains starting with these minimal sets, and add together their associated characteristic functions, call it $v_{k}$, say. Notice, if there were more than one set of smallest cardinality, $v_{k}$ is no longer a binary vector, or simple game. To fix this, we subtract off a truncation of the characteristic function associated with our set chains, starting where our vector has a place containing a value more than 1 , being careful that we do not subtract anything from a place with a 1 in it, as this would break our monotonicity. We continue this process, for the vector $v-v_{k}$, updating $v_{k}$ as we proceed, and after finitely many steps $v=v_{k}$ and we are done.

Definition 3.1 (Axiom 2.2, redux). Assuming Axiom 3.2, An allocation is reasonable for superadditive characteristic functions if

$$
\min _{S, i \notin S}\{v(S \cup\{i\})-v(S)\} \leq \phi_{i}(N ; v) \leq \max _{S, i \notin S}\{v(S \cup\{i\})-v(S)\}
$$

is satisfied for all superadditive $v$.

Note. Recall for an allocation $\phi$ with matrix $A$, we have $\phi_{i}(N ; v)=A_{i} \cdot v$.

Proposition 3.2. If a map is efficient for all superadditive characteristic functions $v$, and we assume Axiom 3.2 holds, then it is efficient for all monotone characteristic functions.

\footnotetext{
${ }^{5}$ This is not one of our previously named superadditive characteristic functions.
} 
Proof. We prove this by viewing the allocation as a matrix. To deal with efficiency, we look at the sums of the column elements, and ensure they add up to

$$
(-1,0, \ldots, 0,1)
$$

following our note following Lemma 2.1. This, as seen in Lemma 2.1, depends on this being true for all $v$, which we can shorten to monotone $v$ thanks to our prior work. This time, however, we can consider only superadditive $v$ via Proposition 3.1. So, following the results in the proof of Lemma 2.1, we obtain all the sums of column elements except the first -1 . This -1 is given to us by the assumption that the row-wise sum is 0 . Hence, as the sum of all the rows, save the first element in each row is 1 , this forces the entries in the first column to sum to -1 . Thus, we have efficiency via the superadditive $v$ only.

If we have a matrix of a reasonable allocation, we can also find the pairings while checking only the superadditive vectors by the following proposition.

Proposition 3.3. Given an allocation $\phi$, reasonable for superadditive characteristic functions, with matrix A, and assuming Axiom 3.2, the row-wise pairing in the matrix can be determined by using only superadditive characteristic functions.

Proof. Certainly, as we have seen previously, the row-wise pairing of elements can be determined by superadditive characteristic functions and their truncations, save the

$$
A_{i, \varnothing}=-A_{i,\{i\}}
$$

pair. Thus, we need only check a subset of the superadditive characteristic functions to obtain all but the $n$ pairings mentioned in Equation (11). Following our prior method of proof, we need the vector $[1,1, \ldots, 1,1]^{t}$ to obtain the last pairings above. This is not superadditive, as $v(\varnothing)>0$. However, this vector is simply a convenient way to ensure that the sum of each row is 0. Supposing Axiom 3.2 holds, observe that the sum of all of the elements in the row is 0 . However, all of the other elements in each row sum to 0 in pairs, except $A_{i, \varnothing}$ and $A_{i,\{i\}}$. Thus, we immediately gain the final pairing, for when we take the row sum, it collapses to the two elements,

$$
A_{i, \varnothing}+A_{i,\{i\}}=0,
$$

we need only re-arrange and obtain the final pairings,

$$
A_{i, \varnothing}=-A_{i,\{i\}}
$$

Note. The pairing alone is not sufficient to show reasonableness, we would additionally need that

$$
\sum_{S} A_{i, S \cup\{i\}}=1
$$

for $S$ without $i$ and $A_{i, S \cup\{i\}} \geq 0$ for the same $S$. Then, certainly $A$ is reasonable.

Notice, with no modifications whatsoever that Lemma 2.20 holds. Additionally, with the background above, we have Theorem 2.22 as well, replacing reasonable with reasonable for superadditive characteristic functions, as the argument does not depend on superadditive or monotone $v$ in the slightest. 
Proposition 3.4. If $\phi$ is reasonable and efficient for superadditive characteristic functions, then it is a convex combination of the special allocations.

Proof. This is mainly a direct consequence of Lemma 2.20 and Propositions 3.2 and 3.3. Suppose we have a matrix $A$ of the allocation $\phi$ that is reasonable for superadditive characteristic functions. By Proposition 3.2 we know that the same efficiency constrains are satisfied. Further, by Proposition 3.3 we have the pairings we seek. Finally, via Lemma 2.20 we see that the extreme points of the reasonable, efficient allocations are the special allocations. To complete the result, we apply Theorem 2.22, with the prior results on reasonable for superadditive characteristic functions $v$ and the proof is complete.

With this result, we note the following corollary.

Corollary (to Proposition 3.4). Given an efficient allocation, reasonable for superadditive characteristic functions implies reasonableness.

Indeed. We can trivially observe that if something lies within the convex combination of the special allocations, then it is reasonable by the vanilla version of Theorem 2.22.

Note. This is quite nontrivial. If one attempts to prove this fact from first principles it is difficult, if not impossible.

Proposition 3.5. Assuming Axiom 3.2, reasonableness implies reasonable for superadditive characteristic functions.

Proof. This is trivially the case. If one satisfies reasonableness for all monotone $v$, Equation (10) is certainly satisfied for all superadditive $v$.

We conclude by distilling our results into the following Theorem.

Theorem 3.6. An efficient allocation is reasonable if and only if it is reasonable for superadditive characteristic functions.

\subsection{Further exploration}

We note that our results here can be generalized further, in both directions. Namely, all of the results we have seen can be made less stringent. In all of our reasonability discussions, we have used only a small set of superadditive characteristic functions,

$$
\begin{aligned}
& v_{a}^{S}(T)= \begin{cases}1 & \text { if } S \subset T \\
0 & \text { else }\end{cases} \\
& v_{b}^{S}(T)= \begin{cases}1 & \text { if } S \subsetneq T \backslash\{i\} \\
0 & \text { else }\end{cases} \\
& v_{c}^{S}(T)= \begin{cases}1 & \text { if } S \subsetneq T \\
0 & \text { else }\end{cases}
\end{aligned}
$$


Our results hold if we are reasonable and efficient for the set $\mathcal{V}_{a b c}$ containing all vectors of this type.

Further, as long as our general set of vectors contains this set of vectors, we can establish a version of reasonableness, and obtain the results once again.

\subsection{Concluding remarks}

As we discussed previously, one of the important ideas of cooperative game theory, and more generally, a way to fairly determine power or distribute gains, is the Shapley value. As such, an understanding of the setting surrounding the value, and the axiomatic assumptions is necessary. Within this paper, we discovered in the general, non-probabilistic context, that alternative assumptions still give us a robust structure.

Even without the Shapley value's uniqueness, the structure within gives us some insight on how a reasonable, efficient allocation is constructed. This insight leads us to other possible values, parallel to Shapley's, offering a structure for an alternative way of distributing the gains of collaboration.

\section{References}

[1] Charalambos D. Aliprantis and Kim C. Border. Infinite dimensional analysis. Springer, Berlin, third edition edition, 2006. A hitchhiker's guide.

[2] Adam Bowers and Nigel J. Kalton. An introductory course in functional analysis. Universitext. Springer, New York, 2014. With a foreword by Gilles Godefroy.

[3] Javier Castro, Daniel Gómez, and Juan Tejada. Polynomial calculation of the Shapley value based on sampling. Comput. Oper. Res., 36(5):1726-1730, 2009.

[4] Jacob N. Clark. Shapley-like values without symmetry. PhD thesis, University of Missouri-Columbia, May 2019.

[5] Jean Derks. Decomposition of games with non-empty core into veto-controlled simple games. Operations-Research-Spektrum, 9(2):81-85, Jun 1987.

[6] Jean Derks. A new proof for weber's characterization of the random order values. Mathematical Social Sciences, 49(3):327-334, May 2005.

[7] Shaheen S. Fatima, Michael Wooldridge, and Nicholas R. Jennings. A linear approximation method for the Shapley value. Artificial Intelligence, 172(14):1673-1699, 2008.

[8] Daniel Gómez, Enrique González-Arangüena, Conrado Manuel, Guillermo Owen, Mónica del Pozo, and Juan Tejada. Centrality and power in social networks: a game theoretic approach. Mathematical Social Sciences, 46(1):27-54, 2003.

[9] Michael Maschler, Eilon Solan, and Shmuel Zamir. Game theory. Cambridge University Press, Cambridge, 2013. Translated from Hebrew by Ziv Hellman and edited by Mike Borns. 
[10] Jean-François Mertens. Some other economic applications of the value. In Robert Aumann and Sergiu Hart, editors, Handbook of Game Theory with Economic Applications Volume 3, pages 2185-2201. Elsevier, 2002.

[11] Dov Monderer and Dov Samet. Variations on the shapley value. In Robert Aumann and Sergiu Hart, editors, Handbook of Game Theory with Economic Applications Volume 3, pages 2055-2076. Elsevier, 2002.

[12] Roger B. Myerson. Graphs and cooperation in games. Mathematics of Operations Research, 2(3):225-229, Aug 1977.

[13] Ramasuri Narayanam and Yadati Narahari. A shapley value-based approach to discover influential nodes in social networks. IEEE Trans. Automation Science and Engineering, 8(1):130-147, 2011.

[14] Guillermo Owen. Multilinear extensions of games. Management Sci., 18:64-79, 1971/72.

[15] Panagiotis Papapetrou, Aristides Gionis, and Heikki Mannila. A shapley value approach for influence attribution. Lecture Notes in Computer Science, pages 549-564, 2011.

[16] Lloyd S. Shapley. A value for $n$-person games. In Harold W. Kuhn and Albert W. Tucker, editors, Contributions to the theory of games, vol. 2, Annals of Mathematics Studies, no. 28, pages 307-317. Princeton University Press, Princeton, N. J., 1953.

[17] Lloyd S. Shapley and Martin Shubik. A method for evaluating the distribution of power in a committee system. American Political Science Review, 48(03):787-792, Sep 1954.

[18] Robert J. Weber. Probabilistic values for games. In Alvin E. Roth, editor, The Shapley Value: Essays in Honor of Lloyd S. Shapley, pages 101-119. Cambridge University Press, 1988. 